\title{
Continuous families of divisors, paracanonical systems and a new inequality for varieties of maximal Albanese dimension
}

\author{
MARGARIDA MENDES LOPES \\ RITA PARDINI \\ Gian Pietro Pirola
}

Given a smooth complex projective variety $X$, a line bundle $L$ of $X$ and $v \in H^{1}\left(\mathcal{O}_{X}\right)$, we say that $v$ is $k$-transversal to $L$ if the complex $H^{k-1}(L) \rightarrow H^{k}(L) \rightarrow H^{k+1}(L)$ is exact. We prove that if $v$ is 1 -transversal to $L$ and $s \in H^{0}(L)$ satisfies $s \cup v=0$, then the first order deformation $\left(s_{v}, L_{v}\right)$ of the pair $(s, L)$ in the direction $v$ extends to an analytic deformation.

We apply this result to improve known results on the paracanonical system of a variety of maximal Albanese dimension, due to Beauville in the case of surfaces and to Lazarsfeld and Popa in higher dimension. In particular, we prove the inequality $p_{g}(X) \geq \chi\left(K_{X}\right)+q(X)-1$ for a variety $X$ of maximal Albanese dimension without irregular fibrations of Albanese general type.

$14 \mathrm{C} 20,14 \mathrm{~J} 29,32 \mathrm{G} 10$

\section{Introduction}

The geometry of the divisors of an irregular algebraic variety is richer than in the regular case, due to the existence of nontrivial continuous families of divisors. Some of these families are intrinsically defined, as the paracanonical system introduced below, which is of fundamental importance in the study of irregular varieties of general type: indeed, the interest in the geometry of the paracanonical system has been the motivation leading to the groundbreaking results of Green and Lazarsfeld [6; 7] on generic vanishing.

Here we consider the following situation: let $X$ be a smooth projective variety, let $\lambda$ be a class in the Néron-Severi group $\mathrm{NS}(X)$ and denote by $\operatorname{Pic}^{\lambda}(X)$ the connected component of $\operatorname{Pic}(X)$ that parametrizes isomorphism classes of line bundles with class $\lambda$. The effective divisors with class $\lambda$ are parametrized by a projective variety $\operatorname{Div}^{\lambda}(X)$ and the characteristic map $c_{\lambda}: \operatorname{Div}^{\lambda}(X) \rightarrow \operatorname{Pic}^{\lambda}(X)$ is defined by $D \mapsto \mathcal{O}_{X}(D)$. The fiber of $c_{\lambda}$ over a point $L \in \operatorname{Pic}^{\lambda}(X)$ is naturally isomorphic to the linear system $|L|$. One defines the continuous rank of $\lambda$ (this terminology is due 
to M A Barja) as $\rho(\lambda):=\min \left\{h^{0}(L) \mid L \in \operatorname{Pic}^{\lambda}(X)\right\}:$ if $\rho(\lambda)>0$ there is exactly one irreducible component $\operatorname{Div}^{\lambda}(X)$ main of $\operatorname{Div}^{\lambda}(X)$ that maps surjectively onto $\operatorname{Pic}^{\lambda}(X)$. The variety $\operatorname{Div}^{\lambda}(X)_{\text {main }}$ is called the main component of $\operatorname{Div}^{\lambda}(X)$; its dimension is equal to $\rho(\lambda)+q(X)-1$.

We study the problem of deciding whether the linear system $|L|$ is contained in $\operatorname{Div}^{\lambda}(X)_{\text {main }}$, and the closely related question of deciding whether $|L|$ is a component of $\operatorname{Div}^{\lambda}(X)$ (ie, whether, in the terminology used by Beauville in [2], the system $|L|$ is exorbitant).

To this end, we introduce the notion of $k$-transversality for a vector $v \in H^{1}\left(\mathcal{O}_{X}\right)$ : we say that $v$ is $k$-transversal to $L$ if the complex given by cup product

$$
H^{k-1}(L) \stackrel{\cup v}{\longrightarrow} H^{k}(L) \stackrel{\cup v}{\longrightarrow} H^{k+1}(L)
$$

is exact. We prove the following.

Theorem 1.1 Let $X$ be a smooth projective variety, let $0 \neq L \in \operatorname{Pic}(X)$ and denote by $\lambda \in \mathrm{NS}(X)$ the class of $L$; let $0 \neq s \in H^{0}(L)$ and $v \in H^{1}\left(\mathcal{O}_{X}\right)$ such that $s \cup v=0$, and denote by $D$ the divisor of zeros of $s$. Then

(i) if $v$ is 1 -transversal to $L$, then $D$ belongs to the closure of $\operatorname{Div}^{\lambda}(X) \backslash|L|$;

(ii) if $v$ is $k$-transversal to $L$ for every $k>0$ and $\chi(L)>0$, then $\rho(L)=\chi(L)$ and $D \in \operatorname{Div}^{\lambda}(X)_{\text {main }}$.

The idea of the proof is to construct inductively a formal deformation of the pair $(s, L)$ over Spec $\mathbb{C} \llbracket t \rrbracket$ : the transversality assumption on $v$ allows one to perform the inductive step (cf Proposition 3.1).

We apply Theorem 1.1 to the analysis of the paracanonical system $\operatorname{Div}^{\kappa}(X)$ of a variety $X$ of maximal Albanese dimension, where $\kappa$ is the canonical class. In view of the generic vanishing theorem of Green and Lazarsfeld, Theorem 1.1 translates this problem into some nontrivial linear and multilinear algebra questions.

One should observe in this particular case the crucial deformation result Proposition 3.1 (and thus Theorem 1.1) could also be derived by the results in [7, Section 3] via Grothendieck duality; however, we believe that the statement of Theorem 1.1, that relates the vanishing of all higher obstructions with the 1-transversality property, will prove useful for the analysis of other situations.

It is traditional to denote the paracanonical system $\operatorname{Div}^{\kappa}(X)$ by $\mathcal{P}_{X}$, or simply by $\mathcal{P}$. The generic vanishing theorem of [6] implies that the continuous rank $\rho(\kappa)$ of $\mathcal{P}$ is equal to the Euler characteristic $\chi\left(K_{X}\right)$, hence for $\chi\left(K_{X}\right)>0$ the paracanonical system $\mathcal{P}$ 
has a main component $\mathcal{P}_{\text {main }}$. Paracanonical systems and conditions for $\left|K_{X}\right|$ to be exorbitant or not have been studied in [2] in the case of surfaces, and by Lazarsfeld and Popa in [8] in the general case. Here we make some further progress in the understanding of paracanonical systems. In dimension greater than 2 , we prove the following result (see Section 2 for the definitions of Albanese general type fibration and $\left.V_{k}(X)\right)$.

Theorem 1.2 Let $n \geq 3$ be an integer and let $X$ be a smooth projective $n$-dimensional variety with irregularity $q \geq n+1$. If $X$ has no Albanese general type fibration, then $\chi\left(K_{X}\right) \geq q-n>0$ and

(i) $p_{g}(X) \geq \chi\left(K_{X}\right)+q-1$;

(ii) $p_{g}(X)=\chi\left(K_{X}\right)+q-1$ if and only if $\left|K_{X}\right| \subset \mathcal{P}_{\text {main }}$;

(iii) if $p_{g}(X)=\chi\left(K_{X}\right)+q-1$, then $q=n+1$;

(iv) if, in addition, $0 \in V_{k}(X)$ is an isolated point for $k>0$, then we have that $p_{g}(X)=\chi\left(K_{X}\right)+q-1$ if and only if $q=n+1$ and $\left(\begin{array}{c}h(X) \\ n\end{array}\right) \equiv 1 \bmod 2$, where $h(X):=\sum_{j=0}^{\lfloor(n-1) / 2\rfloor} h^{0, n-1-2 j}(X)$.

Notice that any smooth ample divisor $X$ in an abelian variety satisfies the equation $p_{g}(X)=\chi\left(K_{X}\right)+q(X)-1$ (cf Example 5.3). In Section 5 we give examples that show that neither the assumption $q(X) \geq \operatorname{dim} X+1$ nor the assumption that $X$ does not have fibrations of Albanese general type can be removed from Theorem 1.2.

To our knowledge, the inequality in Theorem 1.2 had not been conjectured before, possibly due to the fact that for a surface $X$ one has that $p_{g}(X)=\chi\left(K_{X}\right)+q-1$. Indeed, [8, Proposition 5.5] gives necessary and sufficient numerical conditions (that we use in the proof of Theorem 1.2(iv)) in order that $\left|K_{X}\right|$ be exorbitant, assuming the converse inequality $p_{g}(X) \leq \chi\left(K_{X}\right)+q-1$ (which clearly holds when $\left|K_{X}\right|$ is not exorbitant).

Then we turn to the case of surfaces. In [2] Beauville proved that if $X$ is a surface of general type of irregularity $q \geq 2$ without irrational pencils of genus greater than $q / 2$ then the general canonical curve deforms to first order in a 1-transversal direction if and only if $q$ is odd. This shows that $\left|K_{X}\right|$ is exorbitant for $q$ even and gives strong evidence for the fact that $\left|K_{X}\right|$ is not exorbitant if $q$ is odd. Here, thanks to Theorem 1.1, we are able to complete Beauville's results as follows.

Theorem 1.3 Let $X$ be a surface with $\chi\left(K_{X}\right)>0$ and irregularity $q \geq 2$ without irrational pencils of genus greater than $q / 2$. Then

(i) if $q$ is odd, then $\left|K_{X}\right| \subset \mathcal{P}_{\text {main }}$; 
(ii) if $q$ is even, then $\Sigma:=\left|K_{X}\right| \cap \mathcal{P}_{\text {main }}=\left|K_{X}\right| \cap \overline{\left(\mathcal{P} \backslash\left|K_{X}\right|\right)}$ is a reduced and irreducible hypersurface of degree $q / 2$ of $\left|K_{X}\right|$. If, in addition, $X$ has no irrational pencil of genus greater than 1 , then $\operatorname{Sing}(\Sigma)=\{[s] \in \Sigma \mid \operatorname{rk}(\cup s)<q-2\}$.

As an immediate consequence of Theorem 1.3, we are able to answer in the case of surfaces the question, raised by the authors in [9, Section 7], of what is the relation between the base schemes of $\left|K_{X}\right|$ and $\mathcal{P}_{\text {main }}$. Since for $q \geq 3$ the variety $\left|K_{X}\right| \cap \mathcal{P}_{\text {main }}$ is a nondegenerate subvariety of $\left|K_{X}\right|$, we have the following.

Corollary 1.4 Let $X$ be a surface of general type that has no irrational pencil of genus greater than $q / 2$. If $X$ has irregularity $q \geq 3$, then the base scheme of $\mathcal{P}_{\text {main }}$ is contained in the base scheme of $\left|K_{X}\right|$.

Theorems 1.2 and 1.3 imply immediately necessary conditions for the irreducibility of $\mathcal{P}$.

Corollary 1.5 Let $X$ be a smooth projective $n$-dimensional variety with irregularity $q \geq n+1$ that has no Albanese general type fibration. If the paracanonical system $\mathcal{P}_{X}$ is irreducible, then $X$ is one of the following:

(a) a curve;

(b) a surface with $q$ odd;

(c) a variety of dimension $n \geq 3$ with $p_{g}(X)=\chi\left(K_{X}\right)+q-1$ (and $q=n+1$ ).

Notation and conventions We work over the complex numbers; all varieties are projective. If $X$ is a smooth projective variety, we denote as usual by $p_{g}(X)$ the geometric genus $h^{0}\left(K_{X}\right)=h^{n}\left(\mathcal{O}_{X}\right)$, and by $q(X)$ the irregularity $h^{0}\left(\Omega_{X}^{1}\right)=h^{1}\left(\mathcal{O}_{X}\right)$. Recall that by Hodge theory there is an antilinear isomorphism $H^{0}\left(\Omega_{X}^{1}\right) \rightarrow H^{1}\left(\mathcal{O}_{X}\right)$ that we denote by $\alpha \mapsto \bar{\alpha}$. We use the standard notation $\operatorname{Pic}(X)$, resp. $\operatorname{NS}(X)$, for the group of divisors modulo linear, resp. algebraic, equivalence; given $L \in \operatorname{Pic}(X)$, we denote by $\chi(L)$ its Euler characteristic.

For $V$ a complex vector space and $r \geq 1$ an integer, $\mathbb{G}(r, V)$ denotes the Grassmannian of $r$-dimensional vector subspaces of $V$.

Acknowledgments We wish to thank Mihnea Popa for some useful mathematical communications.

The first author is a member of the Center for Mathematical Analysis, Geometry and Dynamical Systems (IST/UTL). The second and the third author are members of GNSAGA-INdAM. This research was partially supported by FCT (Portugal) through program POCTI/FEDER and Project PTDC/MAT/099275/2008 and by MIUR (Italy) through PRIN 2008 "Geometria delle varietà algebriche e dei loro spazi di moduli" and PRIN 2009 "Spazi di moduli e Teoria di Lie." 


\section{Preliminaries}

We recall several known results on irregular varieties that are used repeatedly throughout the paper.

\subsection{Albanese dimension and irrational fibrations}

Let $X$ be a smooth projective variety of dimension $n$. The Albanese dimension $\operatorname{albdim}(X)$ is defined as the dimension of the image of the Albanese map of $X$; in particular, $X$ has maximal Albanese dimension if its Albanese map is generically finite onto its image and it is of Albanese general type if in addition $q(X)>n$. For a normal variety $Y$, we define the Albanese variety $\operatorname{Alb}(Y)$ and all the related notions by considering any smooth projective model of $Y$.

An irregular fibration $f: X \rightarrow Y$ is a morphism with positive dimensional connected fibers onto a normal variety $Y$ with albdim $Y=\operatorname{dim} Y>0$; the map $f$ is called an Albanese general type fibration if in addition $Y$ is of Albanese general type. If $\operatorname{dim} Y=1$, then $Y$ is a smooth curve of genus $b>0$; in that case, $f$ is called an irrational pencil of genus $b$ and it is an Albanese general type fibration if and only if $b>1$.

Notice that if $q(X) \geq n$ and $X$ has no Albanese general type fibration, then $X$ has maximal Albanese dimension.

The so-called generalized Castelnuovo-de Franchis Theorem (see Catanese [3, Theorem 1.14] and Ran [11]) shows how the existence of Albanese general type fibrations is detected by the cohomology of $X$.

Theorem 2.1 (Catanese, Ran) The smooth projective variety $X$ has an Albanese general type fibration $f: X \rightarrow Y$ with $\operatorname{dim} Y \leq k$ if and only if there exist independent 1 -forms $\omega_{0}, \ldots, \omega_{k} \in H^{0}\left(\Omega_{X}^{1}\right)$ such that $\omega_{0} \wedge \omega_{1} \wedge \cdots \wedge \omega_{k}=0 \in H^{0}\left(\Omega_{X}^{k+1}\right)$.

A closely related result, due to Green and Lazarsfeld, is recalled in the next section (Theorem 2.5).

\subsection{Generic vanishing}

Let $X$ be a projective variety of dimension $n$ and let $L \in \operatorname{Pic}(X)$; the $i^{\text {th }}$ cohomological support locus of $L$ is defined as $V_{i}(L, X):=\left\{\eta \mid h^{i}(L+\eta)>0\right\} \subseteq \operatorname{Pic}^{0}(X), i=$ $0, \ldots, n$. The cohomological support loci are closed by the semicontinuity theorem. 
One says that generic vanishing holds for $L$ if $V_{i}(L, X)$ is a proper subvariety of $\operatorname{Pic}^{0}(X)$ for $i>0$.

We identify, as usual, the tangent space to $\operatorname{Pic}^{0}(X)$ at any point with $H^{1}\left(\mathcal{O}_{X}\right)$, so we regard the elements of $H^{1}\left(\mathcal{O}_{X}\right)$ as tangent directions. Given $0 \neq v \in H^{1}\left(\mathcal{O}_{X}\right)$, the derivative complex of $L$ in the direction $v$ is

$$
0 \rightarrow H^{0}(L) \stackrel{\cup v}{\longrightarrow} H^{1}(L) \stackrel{\cup v}{\longrightarrow} \cdots \stackrel{\cup v}{\longrightarrow} H^{n}(L) \rightarrow 0 .
$$

We say that $v$ is $k$-transversal to $L$ if the $k^{\text {th }}$ cohomology group of the complex (2-1) vanishes. This terminology is explained by the following key result (cf [6, Section 1], in particular Theorem 1.6, and [7, Corollary 3.3]).

Proposition 2.2 (Green-Lazarsfeld) Let $X$ be a smooth projective variety and let $L \in \operatorname{Pic}(X)$; consider a point $\eta \in V_{k}(L, X)$ and a nonzero direction $v \in H^{1}\left(\mathcal{O}_{X}\right)$. Then

(i) if $v$ is $k$-transversal to $L+\eta$ then $v$ is not in the tangent cone to $V_{k}(L, X)$ at $\eta$;

(ii) if $\eta \in V_{k}(L, X)$ is general, then either $v$ is $k$-transversal to $L+\eta$ or both maps in the sequence

$$
H^{k-1}(L+\eta) \stackrel{\cup v}{\longrightarrow} H^{k}(L+\eta) \stackrel{\cup v}{\longrightarrow} H^{k+1}(L+\eta)
$$

vanish.

Proposition 2.2 has the following immediate consequence (in fact, the generic vanishing theorem for $K_{X}$ was first proven in [6] using this argument).

Corollary 2.3 Let $X$ be a smooth projective variety and let $L \in \operatorname{Pic}^{0}(X)$; if there exists $\eta \in \operatorname{Pic}^{0}(X)$ and $v \in H^{1}\left(\mathcal{O}_{X}\right)$ such that $v$ is $k$-transversal to $L+\eta$ for every $k>0$, then generic vanishing holds for $L$.

When $L=K_{X}$, we omit $L$ from the notation and simply write $V_{i}(X)$; the loci $V_{i}(X)$ are also called generic vanishing loci of $X$. We recall the main facts about them.

Theorem 2.4 (Green-Lazarsfeld, Simpson) Assume that $X$ has maximal Albanese dimension. Then

(i) $V_{i}(X)$ has codimension greater than or equal to $i$ in $\operatorname{Pic}^{0}(X)$ for $i=0, \ldots, n$;

(ii) $V_{0}(X) \supseteq V_{1}(X) \supseteq \cdots \supseteq V_{n}(X)=\{0\}$;

(iii) the components of $V_{i}(X)$ are translates of complex subtori of $\mathrm{Pic}^{0}(X)$ by torsion points. 
Proof Part (i) is [6, Theorem 2.10], (ii) is from Ein and Lazarsfeld [5, Lemma 1.8]. The fact that the components of $V_{i}(X)$ are translates of subtori is the main result of [7]. Finally, the fact that they are translates by torsion points is proven by Simpson in [12].

For $L$ algebraically equivalent to $K_{X}$, the condition that $v$ is $k$-transversal to $L$ is related to the existence of irregular fibrations, as shown by the following result ([7, Theorem 5.3]), that can also be regarded as a generalization of the Castelnuovo-de Franchis theorem.

Theorem 2.5 (Green-Lazarsfeld) Let $X$ be a smooth projective variety of maximal Albanese dimension, let $\eta \in \operatorname{Pic}^{0}(X)$, let $0 \neq v \in H^{1}\left(\mathcal{O}_{X}\right)$ and set $\omega:=\bar{v} \in H^{0}\left(\Omega_{X}^{1}\right)$. If $v$ is not $k$-transversal to $K_{X}+\eta$, then there exists an irregular fibration $f: X \rightarrow Y$ with $\operatorname{dim} Y \leq n-k$ such that $\omega$ is the pullback of a $1-$ form $\alpha \in H^{0}\left(\Omega_{\operatorname{Alb}(Y)}^{1}\right)$.

Proposition 2.2 and Theorem 2.5 for $\eta=0$, give the following.

Corollary 2.6 If $X$ has no irregular fibration, then 0 is an isolated point of $V_{i}(X)$ for every $i>0$.

\section{Proof of Theorem 1.1}

The key step in the proof of Theorem 1.1 is a deformation result for sections of $L$ that we state next. Denote by $R$ the ring $\mathbb{C} \llbracket t \rrbracket$ of formal power series; for $0 \neq v \in H^{1}\left(\mathcal{O}_{X}\right)$, there is a morphism $\phi_{v}$ : Spec $R \rightarrow \operatorname{Pic}^{\lambda}(X)$ defined by $t \mapsto L \otimes \exp (t v)$, where exp: $H^{1}\left(\mathcal{O}_{X}\right) \rightarrow \operatorname{Pic}^{0}(X)$ denotes the exponential mapping. Pulling back via $\phi_{v}$ a Poincaré line bundle on $X \times \operatorname{Pic}(X)$, one obtains a deformation $L_{v}(t)$ of $L$ over Spec $R$ that we call the (formal) straight line deformation of $L$ in the direction $v$.

Proposition 3.1 Let $X$ be a smooth projective variety, let $L \in \operatorname{Pic}(X)$ be a line bundle and let $s \in H^{0}(L)$ be a nonzero section. If $v \in H^{1}\left(\mathcal{O}_{X}\right)$ is 1 -transversal to $L$ and $s \cup v=0$, then the formal straight line deformation $L_{v}(t)$ of $L$ in the direction $v$ can be lifted to a deformation $\left(s_{v}(t), L_{v}(t)\right)$ of the pair $(s, L)$.

Granting Proposition 3.1 for the moment, we prove Theorem 1.1.

Proof of Theorem 1.1 (i) By Proposition 3.1, there exists a formal deformation $\left(s_{v}(t), L_{v}(t)\right)$ of the pair $(s, L)$ over $\operatorname{Spec} R$, where $L_{v}(t)$ is the straight line deformation of $L$ in the direction $v$. By Artin's convergence Theorem [1, Theorem 1.2], 
there exists an analytic deformation $(\widetilde{s}(t), \widetilde{L}(t))$ of $(s, L)$ over a small disk around $0 \in \mathbb{C}$ such that $\left(s_{v}(t), L_{v}(t)\right)=(\widetilde{s}(t), \widetilde{L}(t)) \bmod t^{2}$.

(ii) Since $v$ is $k$-transversal, $L$ satisfies generic vanishing by Corollary 2.3, hence $\rho(L)=\chi(L)>0$. To prove the claim, it is enough to show that for $t \neq 0$ small the analytic deformation $(\widetilde{s}(t), \widetilde{L}(t))$ is not contained in an irreducible component $Z$ of $\operatorname{Div}^{\lambda}(X)$ different from the main component. By the semicontinuity of the dimension of cohomology groups, it follows that the image in $\operatorname{Pic}^{\lambda}(X)$ of such a component is contained in $L+V_{k}(L, X)$ for some $k>0$. By Proposition 2.2, the vector $v$, being $k$-transversal to $L$, is not in the tangent cone to $L+V_{k}(L, X)$ at the point $L \in \operatorname{Pic}^{\lambda}(X)$, hence $\widetilde{L}(t) \notin L+V_{k}(L, X)$ for $t \neq 0$ small.

The rest of the section is devoted to the proof of Proposition 3.1. We need to state and prove some technical results. Let $D$ be the divisor of zeros of $s$; for $n \geq 1$ consider the twisted restriction sequence

$$
0 \rightarrow \mathcal{O}_{X}((n-1) D) \rightarrow \mathcal{O}_{X}(n D) \rightarrow \mathcal{O}_{D}(n D) \rightarrow 0
$$

and denote by $d_{n}: H^{0}\left(\mathcal{O}_{D}(n D)\right) \rightarrow H^{1}\left(\mathcal{O}_{X}((n-1) D)\right)$ the induced maps in cohomology.

We use Dolbeault cohomology. We consider the sheaf $\mathcal{C}^{\infty}(L)$ of $C^{\infty}$ sections of the line bundle $L$ and we set $\mathcal{C}^{\infty}(L)_{D}:=\mathcal{C}^{\infty}(L) / \mathcal{C}^{\infty}$; in addition, we denote by $\mathcal{A}^{0, q}$ and $\mathcal{A}^{0, q}(L)$ the sheaves of $C^{\infty}(0, q)$-forms and $C^{\infty}(0, q)$-forms with values in $L$, respectively. We have a commutative diagram

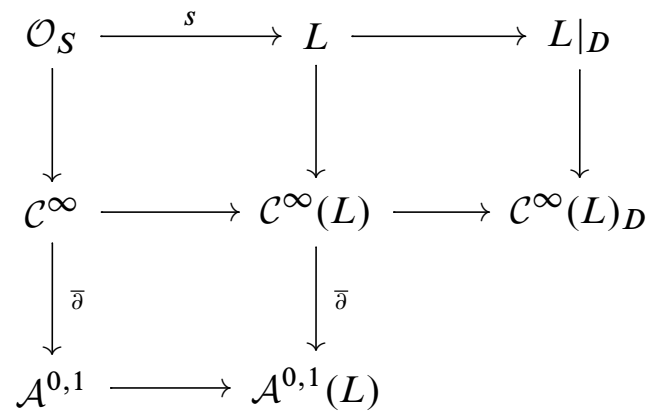

Lemma 3.2 Let $k \geq 1$ be an integer, let $\sigma \in H^{0}\left(\left.L\right|_{D}\right)$ and set $v:=d_{1} \sigma$, let $\psi \in \mathcal{C}^{\infty}(L)$ be a lift of $\sigma$ and let $\zeta \in \mathcal{C}^{\infty}(L)$.

If $v$ is 1 -transversal to $L$ and $\psi^{k} \bar{\partial} \zeta \in \mathcal{A}^{0,1}((k+1) L)$ is $\bar{\partial}$-closed, then $\psi^{k} \bar{\partial} \zeta$ is $\bar{\partial}-$ exact. 
Proof Since $0=\bar{\partial}\left(\psi^{k} \bar{\partial} \zeta\right)=k \psi^{k-1} \bar{\partial} \psi \wedge \bar{\partial} \zeta$, we have $\bar{\partial} \psi \wedge \bar{\partial} \zeta=0$ on the support of $\psi$. On the other hand, $\bar{\partial} \psi=0$ outside the support of $\psi$, hence $\bar{\partial} \psi \wedge \bar{\partial} \zeta=0$ on all of $X$ and therefore $\zeta \bar{\partial} \psi \in \mathcal{A}^{0,1}(L)$ is closed. Since the class $v \in H^{1}\left(\mathcal{O}_{X}\right)$ is represented by $\bar{\partial} \psi \in \mathcal{A}^{0,1}$ and $\bar{\partial} \psi \wedge \zeta \bar{\partial} \psi=0$, we have $v \cup[\zeta \bar{\partial} \psi]=0$. By the assumption $v$ is 1 -transversal to $L$, there exists $g \in H^{0}(L)$ and $\alpha \in \mathcal{C}^{\infty}(L)$ such that

$$
\zeta \bar{\partial} \psi=g \bar{\partial} \psi+\bar{\partial} \alpha=\bar{\partial}(g \psi+\alpha) .
$$

Then we have

$$
\psi^{k} \bar{\partial} \zeta=\bar{\partial}\left(\psi^{k} \zeta\right)-k \psi^{k-1} \zeta \bar{\partial} \psi=\bar{\partial}\left(\psi^{k} \zeta\right)-k \psi^{k-1} \bar{\partial}(g \psi+\alpha) .
$$

Since $k \psi^{k-1} \bar{\partial}(g \psi)=\bar{\partial}\left(\psi^{k} g\right)$, we get

$$
\psi^{k} \bar{\partial} \zeta=\bar{\partial}\left(\psi^{k}(\zeta-g)\right)-k \psi^{k-1} \bar{\partial} \alpha .
$$

For $k=1,(3-2)$ is precisely the claim, and for $k>1$ it gives the inductive step.

Lemma 3.3 Let $\sigma \in H^{0}\left(\mathcal{O}_{D}(D)\right)$ and set $v:=d_{1} \sigma$. Then

(i) $v \cup d_{2} \sigma^{2}=0$;

(ii) if $\xi \in H^{0}(L)$ is such that $d_{2} \sigma^{2}=v \cup 2 \xi$, then $d_{2}\left(\sigma-r_{1}(\xi)\right)^{2}=0$, where $r_{1}: H^{0}(L) \rightarrow H^{0}\left(\mathcal{O}_{D}(D)\right)$ is the restriction map;

(iii) if $d_{2} \sigma^{2}=0$ and $v$ is 1 -transversal to $L$, then $d_{n} \sigma^{n}=0$ for every $n \geq 2$.

Proof (i) We regard $\sigma$ as a global section of $\mathcal{C}^{\infty}(L)_{D}$ and we lift it to a global section $\psi \in \mathcal{C}^{\infty}(L)$. Then $\bar{\partial} \psi$ represents the class $v=d_{1} \sigma \in H^{1}\left(\mathcal{O}_{X}\right)$ and $\bar{\partial} \psi^{2}=2 \psi \bar{\partial} \psi$ represents the class $d_{2} \sigma^{2}$. But then (i) follows, since

$$
v \cup d_{2} \sigma^{2}=[2 \psi \bar{\partial} \psi \wedge \bar{\partial} \psi]=0 .
$$

(ii) The function $\psi-\xi$ is a $\mathcal{C}^{\infty}$ lift of $\sigma-r_{1}(\xi)$, hence $d_{2}\left(\sigma-r_{1}(\xi)\right)^{2}$ is represented by $2(\psi-\xi) \bar{\partial} \psi$. Since, as shown in the proof of (i), $\bar{\partial} \psi$ represents $v$ and $2 \psi \bar{\partial} \psi$ represents $d_{2} \sigma^{2}$, we have $[2(\psi-\xi) \bar{\partial} \psi]=d_{2} \sigma^{2}-v \cup 2 \xi=0$.

(iii) Since $d_{2} \sigma^{2}=0$, there exists $\zeta \in \mathcal{C}^{\infty}(L)$ such that $\psi \bar{\partial} \psi=\bar{\partial} \zeta$. For $n \geq 2$ the class $d_{n} \sigma^{n}$ is represented by $\bar{\partial}\left(\psi^{n}\right)=n \psi^{n-1} \bar{\partial} \psi=n \psi^{n-2} \bar{\partial} \zeta$, hence it vanishes by Lemma 3.2 since $v$ is 1 -transversal to $L$.

Proof of Proposition 3.1 Denote by $D$ the divisor of zeros of $s$ and for $n \geq 1$ let $r_{n}: H^{0}\left(\mathcal{O}_{X}(n D)\right) \rightarrow H^{0}\left(\mathcal{O}_{D}(n D)\right)$ be the restriction map. Let $\left\{U_{i}\right\}$ be an open cover of $X$ consisting of polydiscs, denote by $g_{i j} \in \mathcal{O}_{X}^{*}\left(U_{i j}\right)$ a system of transition functions for $L=\mathcal{O}_{X}(D)$, by $s_{i} \in \mathcal{O}_{X}\left(U_{i}\right)$ local representations of $s$ and by $a_{i j} \in \mathcal{O}_{X}\left(U_{i j}\right)$ a cocycle that represents $v$. 
Set $V_{i}:=U_{i} \times \operatorname{Spec} R$; transition functions for $L_{v}(t)$ with respect to the open cover $\left\{V_{i}\right\}$ of $X \times \operatorname{Spec} R$ are given by

$$
g_{i j}(t)=g_{i j} \exp \left(t a_{i j}\right)=g_{i j}\left(1+a_{i j} t+\frac{a_{i j}^{2} t^{2}}{2}+\cdots+\frac{a_{i j}^{n} t^{n}}{n !}+\cdots\right) .
$$

We look for functions $s_{i}(t) \in \mathcal{O}_{X \times \operatorname{Spec} R}\left(V_{i}\right)$ that satisfy

$$
s_{i}(t)=g_{i j}(t) s_{j}(t)
$$

We write formally $s_{i}(t)=s_{i} \exp \left(\sigma_{i} t+\sum_{r \geq 2} \tau_{i}^{(r)} t^{r}\right)$, with $\sigma_{i}, \tau_{i}^{(r)}$ meromorphic functions on $U_{i}$ with poles only on $D$, and we start by solving (3-4) $\bmod t^{2}$, namely we look for functions $\sigma_{i} \in \mathcal{O}_{X}(D)\left(U_{i}\right)$ that on $U_{i j}$ satisfy

$$
\sigma_{i}=a_{i j}+\sigma_{j}
$$

Equivalently, we look for a section $\sigma \in H^{0}\left(\mathcal{O}_{D}(D)\right)$ such that $d_{1} \sigma=v$. Consider the long cohomology sequence associated with the standard restriction sequence on for $D$ :

$$
\cdots \rightarrow H^{0}\left(\mathcal{O}_{X}(D)\right) \stackrel{r_{1}}{\rightarrow} H^{0}\left(\mathcal{O}_{D}(D)\right) \stackrel{d_{1}}{\longrightarrow} H^{1}\left(\mathcal{O}_{X}\right) \stackrel{\cup s}{\longrightarrow} H^{1}\left(\mathcal{O}_{X}(D)\right) \rightarrow \cdots
$$

Since $s \cup v=0$ by assumption, Equation (3-5) can be solved, and any two solutions differ by a section of $H^{0}\left(\mathcal{O}_{X}(D)\right)$. The second step is to solve

$$
\tau_{i}^{(2)}=\tau_{j}^{(2)} \text { on } U_{i j}, \quad \tau_{i}^{(2)}+\sigma_{i}^{2} / 2 \in \mathcal{O}_{X}(D)\left(U_{i}\right),
$$

for every $i, j$. Namely, we look for a section $\tau^{(2)} \in H^{0}\left(\mathcal{O}_{X}(2 D)\right)$ that lifts the section $-\sigma^{2} / 2 \in H^{0}\left(\mathcal{O}_{D}(2 D)\right)$. Hence we must have that $d_{2} \sigma^{2}=0$. By Lemma 3.3(i), $v \cup d_{2} \sigma^{2}=0$, hence by the assumption that $v$ is transversal to $L$ there exists $\xi \in H^{0}\left(\mathcal{O}_{X}(D)\right)$ such that $d_{2} \sigma^{2}=v \cup 2 \xi$. Hence we have $d_{1}\left(\sigma-r_{1}(\xi)\right)=d_{1} \sigma=v$ and by Lemma 3.3(ii) we have $d_{2}\left(\sigma-r_{1}(\xi)\right)^{2}=0$.

Therefore we may replace $\sigma$ by $\sigma-r_{1}(\xi)$ and then solve (3-6). The third step consists in solving

$$
\tau_{i}^{(3)}=\tau_{j}^{(3)} \text { on } U_{i j}, \quad \tau_{i}^{(3)}+\sigma_{i} \tau_{i}^{(2)}+\frac{\sigma_{i}^{3}}{3} \in \mathcal{O}_{X}(D)\left(U_{i}\right),
$$

for every $i, j$. As in the previous step, (3-7) has a solution $\tau^{(3)} \in H^{0}\left(\mathcal{O}_{X}(3 D)\right)$ if and only if

$$
d_{3}\left(3 \sigma r_{2}\left(\tau^{(2)}\right)+\sigma^{3}\right)=0
$$

Since by construction $r_{2}\left(\tau^{(2)}\right)$ is a nonzero multiple of $\sigma^{2}$, this is equivalent to $d_{3} \sigma^{3}=0$. Hence (3-7) can be solved by Lemma 3.3(iii). By the same argument, one shows inductively the existence of $\tau^{(r)}$ for every $r \geq 4$. 


\section{Paracanonical systems}

\subsection{Proof of Theorem 1.2}

We let $X$ be a smooth projective variety with albdim $X=\operatorname{dim} X=n \geq 2$; for the sake of brevity, we write $p_{g}, q, \chi$ instead of $p_{g}(X), q(X), \chi\left(K_{X}\right)$. We assume that $\chi>0$, so that we can consider the main paracanonical system.

We write $\mathbb{P}:=\mathbb{P}\left(H^{1}\left(\mathcal{O}_{X}\right)\right)$ and define an incidence subvariety $\mathcal{I} \subset \mathbb{P} \times\left|K_{X}\right|$ as follows:

$$
\mathcal{I}:=\{([v],[s]) \mid v \cup s=0\} .
$$

Let $\mathcal{I}_{\text {main }} \subseteq \mathcal{I}$ be the closure of the open subset consisting of pairs ([v], $[s]$ ) such that $v$ is 1-transversal to $K_{X}$. We consider the two projections of $\mathbb{P} \times\left|K_{X}\right|$ and we set $\Sigma \subseteq\left|K_{X}\right|$ and respectively $\Sigma_{\text {main }} \subseteq\left|K_{X}\right|$, the image of $\mathcal{I}$, respectively $\mathcal{I}_{\text {main }}$, via the second projection. Hence $\Sigma$ is the locus of canonical divisors that deform to first order in some nonzero direction $v$, and $\Sigma_{\text {main }}$ is the closure of the locus of canonical divisors that deform to first order in some direction $v$ that is 1 -transversal to $K_{X}$. The key observation is the following.

\section{Lemma 4.1 One has inclusions}

$$
\Sigma_{\text {main }} \subseteq \mathcal{P}_{\text {main }} \cap\left|K_{X}\right| \subseteq\left|K_{X}\right| \cap \overline{\left(\mathcal{P} \backslash\left|K_{X}\right|\right)} \subseteq \Sigma .
$$

Proof Let $v \in H^{1}\left(\mathcal{O}_{X}\right)$ be a vector. If $v$ is $k$-transversal to $K_{X}$, then $v$ is not in the tangent cone to $V_{k}(X)$ at 0 by Proposition 2.2(i); on the other hand, if $v$ is not $k$-transversal to $K_{X}$, then it is not in the tangent cone to $V_{k}(X)$ at 0 by [7, Corollary 3.3]. Summing up, $v$ is $k$-transversal to $K_{X}$ if and only if it is not in the tangent cone to $V_{k}(X)$ at 0 . Since by Theorem 2.4(ii) $V_{1}(X) \supseteq V_{k}(X)$ for every $k \geq 1, v$ is 1 -transversal to $K_{X}$ if and only if it is $k$-transversal to $K_{X}$ for every $k \geq 1$. Hence the first inclusion is a consequence of Theorem 1.1(ii). The second one is obvious.

To prove the last one, consider $D=(s) \notin \Sigma$; the sequence

$$
0 \rightarrow H^{0}\left(\mathcal{O}_{X}\right) \rightarrow H^{0}\left(\mathcal{O}_{X}(D)\right) \rightarrow H^{0}\left(\mathcal{O}_{D}(D)\right) \rightarrow 0
$$

is exact. Since $H^{0}\left(\mathcal{O}_{D}(D)\right)$ is the tangent space to $\mathcal{P}$ at the point $D$, this means that $\mathcal{P}$ and $\left|K_{X}\right|$ have the same tangent space at $D$. Since $\left|K_{X}\right|$ is a smooth variety, this means that $\left|K_{X}\right|$ and $\mathcal{P}$ coincide near $D$, so $\left|K_{X}\right|$ is an irreducible component of $\mathcal{P}$, ie, it is exorbitant. In particular $D \notin \mathcal{P}_{\text {main }}$.

Now we start to study the geometry of the varieties that we have introduced. 
Lemma 4.2 (i) $\mathcal{I}_{\text {main }}$ is irreducible of dimension $\chi+q-2$;

(ii) if $0 \in V_{k}(X)$ is an isolated point for $k>0$, then $\left|K_{X}\right| \cap \overline{\left(\mathcal{P} \backslash\left|K_{X}\right|\right)}=\Sigma_{\text {main }}$.

Proof Denote by $\operatorname{pr}_{i}, i=1,2$, the restrictions to $\mathcal{I}, \mathcal{I}_{\text {main }}$ of the two projections of $\mathbb{P} \times\left|K_{X}\right|$.

(i) As we have already remarked in the proof of Lemma 4.1, a vector $v \in H^{1}\left(\mathcal{O}_{X}\right)$ is 1 -transversal to $K_{X}$ if and only if it is $k$-transversal to $K_{X}$ for every $k>0$. Hence, if $v \in H^{1}\left(\mathcal{O}_{X}\right)$ is 1 -transversal to $K_{X}$, then $\operatorname{pr}_{1}^{-1}([v])$ is a projective space of dimension $\chi-1$. Since by Theorem 2.5 , the classes $[v]$ such that $v$ is 1 -transversal to $K_{X}$ form a nonempty open subset of $\mathbb{P}\left(H^{1}\left(\mathcal{O}_{X}\right)\right)$, the claim follows immediately.

(ii) By Proposition 2.2, the point $0 \in V_{k}(X)$ is isolated for $k>0$ if and only if every $0 \neq v \in H^{1}\left(\mathcal{O}_{X}\right)$ is $k$-transversal to $K_{X}$ for $k>0$, hence, as explained in the proof of (i), if and only if every $0 \neq v \in H^{1}\left(\mathcal{O}_{X}\right)$ is 1 -transversal to $K_{X}$. Therefore we have $\mathcal{I}=\mathcal{I}_{\text {main }}$, and the claim follows by Lemma 4.1.

Next we need a linear algebra result, which is an infinitesimal version of the so-called Ker/Coker Lemma.

Lemma 4.3 Let $V, W$ be complex vector spaces, let $X \subset \operatorname{Hom}(V, W)$ be an irreducible closed subset and let $f \in X$ be general.

If $g$ is tangent to $X$ at the point $f$, then $g(\operatorname{ker}(f)) \subseteq \operatorname{Im} f$.

Proof By the generality of $f$, we may assume that $X$ is smooth at $f$ and that rk $f$ is equal to the maximum of $\operatorname{rk} h$ for $h \in X$; we set $r:=\operatorname{dim} \operatorname{Ker} f$. Then there is an analytic map $F: \Delta \rightarrow X$, where $\Delta$ is a small disk around $0 \in \mathbb{C}$, such that $F(t)=f+g t+\cdots$; then $t \rightarrow U(t):=\operatorname{ker} F(t)$ defines an analytic map $\Delta \rightarrow \mathbb{G}(r, V)$, where $\mathbb{G}(r, V)$ is the Grassmannian. Given $u \in \operatorname{ker} f$, we can lift $t \rightarrow U(t)$ to the universal family on $\mathbb{G}(r, V)$, namely there exists an analytic map $u: \Delta \rightarrow V$ such that $u(t)=u+u_{1} t+\cdots$ and $F(t) u(t)=0$. Hence $0=\left.(F(t) u(t))^{\prime}\right|_{t=0}=f u_{1}+g u$, ie, $g u=-f u_{1} \in \operatorname{Im} f$.

We can now complete the proof of Theorem 1.2.

Proof of Theorem 1.2 Since $X$ has no Albanese general type fibration and $q \geq n+1$, the inequality $\chi\left(K_{X}\right) \geq q(X)-n$ holds by Pareschi and Popa [10].

(i) Consider the projection $\mathrm{pr}_{2}: \mathcal{I}_{\text {main }} \rightarrow \Sigma_{\text {main }} \subseteq\left|K_{X}\right|$. If for general $[s] \in \Sigma_{\text {main }}$ the fiber $\operatorname{pr}_{2}^{-1}([s])$ is a point, or, equivalently, if the dimension $t$ of the kernel $N$ of 
the map $\cup s: H^{1}\left(\mathcal{O}_{X}\right) \rightarrow H^{1}\left(K_{X}\right)$ is equal to 1 , then the inequality $\chi+q-1 \leq p_{g}$ follows by Lemma 4.2.

So assume $t \geq 2$ and denote by $\mathbb{P}(T) \subseteq\left|K_{X}\right|$ the projective tangent space to $\Sigma_{\text {main }}$ at a general point $[s]$. Let $\alpha, \beta \in H^{0}\left(\Omega_{X}^{1}\right)$ be independent 1 -forms such that $\bar{\alpha}, \bar{\beta} \in N$. Given $s^{\prime} \in T \subset H^{0}\left(K_{X}\right)$, then by Lemma 4.3 there exists $\gamma \in H^{0}\left(\Omega_{X}^{1}\right)$ such that $s^{\prime} \wedge \bar{\alpha}=s \wedge \bar{\gamma}$. Thus we have $s^{\prime} \wedge \bar{\alpha} \wedge \bar{\beta}=s \wedge \bar{\gamma} \wedge \bar{\beta}=-s \wedge \bar{\beta} \wedge \bar{\gamma}=0$, for every $\bar{\alpha}, \bar{\beta} \in N$. It follows $T$ is annihilated by the image $S$ of the map $\bigwedge^{2} N \otimes H^{n-2}\left(\mathcal{O}_{X}\right) \rightarrow H^{n}\left(\mathcal{O}_{X}\right)$. Denote by $\mathbb{G}_{N}$ the subset of the Grassmannian $\mathbb{G}\left(n, H^{1}\left(\mathcal{O}_{X}\right)\right)$ consisting of the $n-$ dimensional vector subspaces of $H^{1}\left(\mathcal{O}_{X}\right)$ that intersect $N$ in dimension greater than or equal to 2 . Since by assumption $X$ has no Albanese general type fibration, by Theorem 2.1 the wedge product induces a finite map $\mathbb{G}_{N} \rightarrow \mathbb{P}(S)$. Hence, we have $\operatorname{codim} \Sigma_{\text {main }}=\operatorname{codim} T \geq \operatorname{dim} S \geq \operatorname{dim} \mathbb{G}_{N}+1=(n-2)(q-n)+2(t-2)+1$ if $q \geq n+t-1$ and codim $\Sigma_{\text {main }} \geq n(q-n)+1$ if $q<n+t-1$. Since we have $\operatorname{dim} \Sigma_{\text {main }}=\operatorname{dim} \mathcal{I}_{\text {main }}-(t-1)=\chi+q-t$, this can be rewritten as

(a) $p_{g}-(\chi+q-t) \geq(n-2)(q-n)+2 t-3$, if $q \geq n+t-1$;

(b) $p_{g}-(\chi+q-t) \geq n(q-n)+1$, if $q<n+t-1$.

In case (a) we obtain $p_{g}-(\chi+q-1) \geq(n-2)(q-n)>0$; in case (b), since $t \leq q$, we get $p_{g}-(\chi+q-1) \geq(n-1)(q-n)-n+2 \geq(n-1)-n+2=1$.

(ii) If $\left|K_{X}\right| \subset \mathcal{P}_{\text {main }}$, then $p_{g} \leq \chi+q-1$ and therefore $p_{g}=\chi+q-1$ by (i). Conversely, the proof of (i) shows that if $p_{g}=\chi+q-1$ we have $t=1$. Hence in this case $\operatorname{dim} \Sigma_{\text {main }}=\chi+q-2$ and therefore $\Sigma_{\text {main }}=\left|K_{X}\right|$ and $\left|K_{X}\right| \subset \mathcal{P}_{\text {main }}$ is not exorbitant.

(iii) By (ii), if $p_{g}=\chi+q-1$, then for general $s \in H^{0}\left(K_{X}\right)$ the kernel of $\cup s: H^{1}\left(\mathcal{O}_{X}\right) \rightarrow H^{1}\left(K_{X}\right)$ is $1-$ dimensional. Let $s \in H^{0}\left(K_{X}\right)$ be general and take $0 \neq \alpha \in H^{0}\left(\Omega_{X}^{1}\right)$ such that $s \wedge \bar{\alpha}=0$. We denote by $W$ the image of the map $H^{0}\left(K_{X}\right) \rightarrow H^{1}\left(K_{X}\right)$ defined by $w \mapsto w \wedge \bar{\alpha}$. By Lemma 4.3, for every $w \in H^{0}\left(K_{X}\right)$ there exists $\beta \in H^{0}\left(\Omega_{X}^{1}\right)$ such that $w \wedge \bar{\alpha}=s \wedge \bar{\beta}$, namely $W$ is contained in the image of the map $\cup s: H^{1}\left(\mathcal{O}_{X}\right) \rightarrow H^{1}\left(K_{X}\right)$. It follows that $W$ has dimension less than or equal to $q-1$.

Let $\bar{M} \subset H^{1}\left(\mathcal{O}_{X}\right)$ be a subspace such that $\langle\bar{\alpha}\rangle \oplus \bar{M}=H^{1}\left(\mathcal{O}_{X}\right)$, and consider $M \subset H^{0}\left(X, \Omega_{X}^{1}\right)$. Now take a decomposable form $s_{1}=\beta_{1} \wedge \cdots \wedge \beta_{n-1} \wedge \alpha$ with $\beta_{i} \in M$ independent. By the assumption that $X$ has no Albanese general type fibration, we have $s_{1} \neq 0$, hence $s_{1} \wedge \bar{s}_{1} \neq 0$, and, a fortiori, $s_{1} \wedge \bar{\alpha} \neq 0$. Hence the map $\wedge^{n-1} M \rightarrow W$ defined by $s \mapsto s \wedge \alpha \wedge \bar{\alpha}$ induces a finite morphism $\mathbb{G}(n-1, M) \rightarrow \mathbb{P}(W)$. It follows that $q-2 \geq(n-1)(q-1-(n-1))=(n-1)(q-n)$, that is $(n-2) q \leq(n-2)(n+1)$ and thus $q \leq n+1$. 
(iv) By (ii) the equality $p_{g}=\chi+q-1$ holds if and only if $\left|K_{X}\right| \subset \mathcal{P}_{\text {main }}$, if and only if, since $0 \in V_{k}(X)$ is isolated for $k>0,\left|K_{X}\right|$ is not exorbitant. In addition, by (iii) if $p_{g}(X)=\chi(X)+q-1$ then $q=n+1$.

Hence, by [8, Proposition 5.5], in our situation $\left|K_{X}\right|$ is not exorbitant if and only if the coefficient $s_{n}$ of $t^{n}$ in the formal power series expansion in $\mathbb{Z} \llbracket t \rrbracket$ of the rational function $\prod_{j=1}^{n}(1+j t)^{(-1)^{j+1} h^{0, n-j}}$ does not vanish.

As explained in [8], $s_{n}$ is the degree in $\mathbb{P}\left(H^{1}\left(\mathcal{O}_{X}\right)\right)$ of a general fiber of the map $\mathcal{I}_{\text {main }} \rightarrow \mathbb{P}\left(H^{0}\left(K_{X}\right)\right)$; since by the proof of (i) this fiber is either empty or a point, it is enough to compute $s_{n}$ modulo 2 . Finally, it is an elementary computation to show that $s_{n} \equiv\left(\begin{array}{c}h(X) \\ n\end{array}\right) \bmod 2$.

\subsection{Proof of Theorem 1.3}

Let $X$ be a surface with irregularity $q \geq 2$ and with $\chi:=\chi\left(K_{X}\right)>0$ that has no irrational pencil of genus greater than $q / 2$. Recall that $p_{g}=\chi\left(K_{X}\right)+q-1$.

As explained in the introduction, part of the results that follow were already proven in [2], but in order to give a clear presentation of our results we prefer to give all the proofs.

Lemma 4.4 The irreducible components of $\mathcal{I}$ distinct from $\mathcal{I}_{\text {main }}$ have dimension less than $p_{g}-1$.

Proof Let $v \in H^{1}\left(\mathcal{O}_{X}\right)$ and let $\alpha=\bar{v} \in H^{0}\left(\Omega_{X}^{1}\right)$ : by Hodge theory $v$ is not $1-$ transversal to $K_{X}$ if and only if the sequence

$$
H^{0}\left(\mathcal{O}_{X}\right) \stackrel{\wedge \alpha}{\longrightarrow} H^{0}\left(\Omega_{X}^{1}\right) \stackrel{\wedge \alpha}{\longrightarrow} H^{0}\left(K_{X}\right)
$$

is not exact. Hence by the classical Castelnuovo-de Franchis Theorem (cf also Theorems 2.1 and 2.5$)$, the set of classes $[v] \in \mathbb{P}:=\mathbb{P}\left(H^{1}\left(\mathcal{O}_{X}\right)\right.$ such that $v$ is not 1-transversal to $\left|K_{X}\right|$ is the union of the (finitely many) mutually disjoint linear subspaces $\mathbb{P}\left(\overline{f^{*} H^{0}\left(K_{B}\right)}\right)$, where $f: X \rightarrow B$ is a pencil of genus $b>1$. Fix such a pencil $f$ and write $W:=f^{*} H^{0}\left(K_{B}\right)$. Let $v=\bar{\alpha}$, with $\alpha \in W$, and choose a subspace $N \subset H^{0}\left(\Omega_{X}^{1}\right)$ such that $H^{0}\left(\Omega_{X}^{1}\right)=W \oplus N$ : again by the Castelnuovo-de Franchis theorem, we have $\alpha \wedge \beta \neq 0$ for every $0 \neq \beta \in N$. Therefore $\int_{X} \alpha \wedge \beta \wedge \bar{\alpha} \wedge \bar{\beta} \neq 0$, hence $\alpha \wedge \beta \wedge \bar{\alpha} \neq 0 \in H^{1}\left(K_{X}\right)$. This shows that the linear map $\cup v: H^{0}\left(K_{X}\right) \rightarrow H^{1}\left(K_{X}\right)$ has rank greater than or equal to $q-b$. Hence $\operatorname{pr}_{1}^{-1}([v])$ has dimension less than or equal to $p_{g}-q+b-1$ and the preimage in $\mathcal{I}$ of $\mathbb{P}\left(\overline{f^{*} H^{0}\left(K_{B}\right)}\right)$ has dimension less than or equal to $p_{g}-q+2 b-2<p_{g}-1$, by the assumption that $b \leq q / 2$. 
Assume that $Z$ is an irreducible component of $\mathcal{I}$ distinct from $\mathcal{I}_{\text {main }}$ and consider the first projection $\operatorname{pr}_{1}: \mathcal{I} \rightarrow \mathbb{P}:$ by the definition of $\mathcal{I}_{\text {main }}, \operatorname{pr}_{1}(Z)$ is contained in $\mathbb{P}\left(\overline{f^{*} H^{0}\left(K_{B}\right)}\right)$ for some irrational pencil $f: X \rightarrow B$, and the claim follows by the previous discussion.

Given a 2 -form $s \in H^{0}\left(K_{X}\right)$, the linear map $\cup s: H^{1}\left(\mathcal{O}_{X}\right) \rightarrow H^{1}\left(K_{X}\right) \cong H^{1}\left(\mathcal{O}_{X}\right)^{\vee}$ induces a skew-symmetric bilinear form on $H^{1}\left(\mathcal{O}_{X}\right)$ (by Serre duality) that we denote by $c_{s}$. Clearly, $\Sigma$ is the subset of classes $[s]$ such that $c_{S}$ is degenerate.

Lemma 4.5 (i) If $q$ is odd, then $\Sigma=\Sigma_{\text {main }}=\left|K_{X}\right|$.

(ii) If $q$ is even, then $\Sigma=\Sigma_{\text {main }}$ is the zero set of a polynomial Pf of degree $q / 2$.

Proof (i) If $q$ is odd, then $c_{s}$, being skew-symmetric, is degenerate for every $s \in H^{0}\left(K_{X}\right)$, hence $\Sigma=\left|K_{X}\right|$. By Lemma $4.4, \mathcal{I}_{\text {main }}$ is the only component of $\mathcal{I}$ that can dominate $\left|K_{X}\right|$, hence we also have $\Sigma_{\text {main }}=\left|K_{X}\right|$.

(ii) For $q$ even, $\Sigma$ is the zero locus of the pull back Pf of the Pfaffian polynomial in $\bigwedge^{2} H^{1}\left(\mathcal{O}_{X}\right)^{\vee}$, hence it is either a divisor or it is equal to $\left|K_{X}\right|$. The latter possibility cannot occur by dimension reasons, since for every $[s] \in \Sigma$ the fiber $\operatorname{pr}_{2}^{-1}([s])$ is an odd dimensional linear space. So $\Sigma$ is a divisor. Let $\Delta \subseteq \Sigma$ be an irreducible component: then $\operatorname{pr}_{2}^{-1}(\Delta)$ has a component of dimension greater than or equal to $p_{g}-1$, hence by Lemma $4.4 \operatorname{pr}_{2}^{-1}(\Delta)$ contains $\mathcal{I}_{\text {main }}$. It follows that $\Delta=\Sigma_{\text {main }}=\Sigma$.

Lemma 4.6 Assume that $q \geq 4$ is even and let $[s] \in \Sigma$ be such that

(a) the map $\cup s: H^{1}\left(\mathcal{O}_{X}\right) \rightarrow H^{1}\left(K_{X}\right)$ has rank $q-2$;

(b) if $\operatorname{ker} \cup s=\langle\bar{\alpha}, \bar{\beta}\rangle$, with $\alpha, \beta \in H^{0}\left(\Omega_{X}^{1}\right)$, then $\alpha \wedge \beta \neq 0$.

Then the hypersurface $Z$ defined by Pf (cf Lemma 4.5) is smooth at $[s]$.

Proof Set $w=\alpha \wedge \beta$; we are going to show that the line $M \subset\left|K_{X}\right|$ that joins $[s]$ and $[w]$ intersects $Z$ with multiplicity 1 at $[s]$. We regard $c_{s}$ and $c_{w}$ as alternating forms on $H^{1}\left(\mathcal{O}_{X}\right)$. Let

$$
J:=\left(\begin{array}{cc}
0 & 1 \\
-1 & 0
\end{array}\right)
$$

it is possible to complete $\alpha, \beta$ to a basis of $H^{1}\left(\mathcal{O}_{X}\right)$ in such a way that the matrices $A, B$ associated to $c_{s}, c_{w}$ have the form

$$
A=\left(\begin{array}{ccccc}
0 & 0 & \cdots & \cdots & 0 \\
0 & J & 0 & \cdots & 0 \\
\vdots & \ddots & \ddots & \ddots & \vdots \\
0 & \cdots & 0 & J & 0 \\
0 & \cdots & \cdots & \cdots & J
\end{array}\right), \quad B=\left(\begin{array}{c|c}
C & -{ }^{t} N \\
\hline N & M
\end{array}\right),
$$


where $M$ is a $(q-2) \times(q-2)$ antisymmetric matrix and $C$ is a $2 \times 2$ antisymmetric matrix. Notice $\operatorname{det} C \neq 0$ by the condition $\alpha \wedge \beta \neq 0$. Set $D(\lambda, \mu):=\operatorname{det}(\lambda A+\mu B)$; then it is easy to see that the coefficient in $D(\lambda, \mu)$ of the monomial $\lambda^{q-2} \mu^{2}$ is equal to $\operatorname{det} C \neq 0$. Hence $\mu=0$ is a double root of $D(\lambda, \mu)$. Since $D(\lambda, \mu)$ is the square of the Pfaffian $\operatorname{Pf}(\lambda, \mu)$ of $\lambda A+\mu B$, it follows that $\mu=0$ is a simple root of $\operatorname{Pf}(\lambda, \mu)$. So the intersection multiplicity of $M$ and $Z$ at $[s]$ is equal to 1 , and thus $Z$ is smooth at $[s]$.

Conclusion of the proof of Theorem 1.3 (i) By Lemma 4.5, we have $\Sigma_{\text {main }}=\left|K_{X}\right|$, hence $\left|K_{X}\right| \subset \mathcal{P}_{\text {main }}$ by Lemma 4.1.

(ii) If $q$ is even, then we have that $\Sigma=\Sigma_{\text {main }}$ by Lemma 4.5, and therefore we have that $\left|K_{X}\right| \cap \mathcal{P}_{\text {main }}=\left|K_{X}\right| \cap \overline{\left(\mathcal{P} \backslash\left|K_{X}\right|\right)}=\Sigma$ by Lemma 4.1. By Lemma 4.5, the closed set $\Sigma$ is irreducible and it is the zero locus of a polynomial Pf of degree $q / 2$. In particular, if $q=2$ then $\Sigma$ is a hyperplane, so we may assume from now on that $q \geq 4$. Counting dimensions, one sees that if $[s] \in \Sigma$ is general, then $\operatorname{pr}_{2}^{-1}([s])$ has dimension 1. This is equivalent to the fact that the linear map $\cup s$ has rank $q-2$. In addition, the kernel of $\cup s$ contains a general $\bar{\alpha} \in H^{1}\left(\mathcal{O}_{X}\right)$, hence $s$ satisfies the assumptions of Lemma 4.6, and so $[s]$ is a smooth point of the hypersurface $Z$ defined by Pf. Thus $\Sigma=Z$ is a reduced and irreducible hypersurface of degree $q / 2$. Finally, if $X$ has no irrational pencil of genus greater than 1 , then every $s \in H^{0}\left(K_{X}\right)$ such that $\cup s$ has rank $q-2$ satisfies the assumptions of Lemma 4.6, hence it corresponds to a smooth point of $\Sigma$. Conversely, an argument similar to the proof of Lemma 4.6 shows that if $\cup s$ has rank less than $q-2$, then $[s] \in \Sigma$ is singular.

\section{Examples and open questions}

The first two examples here show that neither the assumption that $q(X) \geq \operatorname{dim} X+1$ nor the assumption that $X$ has no fibration of Albanese general type, respectively, can be removed from Theorem 1.2; the third one shows that when $q(X)=\operatorname{dim} X+1$ the canonical system $\left|K_{X}\right|$ may or may not be exorbitant.

Example 5.1 (Varieties with $q(X)=\operatorname{dim} X$ and $p_{g}(X)<\chi\left(K_{X}\right)+q-1$ ) Our starting point is the example, constructed by Chen and Hacon in [4, Section 4], of a threefold $Y$ of general type and maximal Albanese dimension with $p_{g}(Y)=1, q=3$ and $\chi\left(K_{Y}\right)=0$, so that $\left.p_{g}(Y)-\left(\chi\left(K_{Y}\right)\right)+q(Y)-1\right)=-1<0$.

Now let $H \in \operatorname{Pic}(Y)$ be very ample, let $D \in|2 H|$ be a smooth divisor and let $p: X \rightarrow Y$ be the double cover given by the relation $2 H \equiv D$. The variety $X$ is a smooth threefold of general type and its Albanese map is surjective. By the functoriality of the Albanese 
map, if $X \rightarrow Z$ is any fibration then the Albanese map of $Z$ is also surjective. In particular, $X$ has no fibration of Albanese general type.

The usual formulae for double covers give

$$
\begin{gathered}
\chi\left(K_{X}\right)=\chi\left(K_{Y}\right)+\chi\left(K_{Y}+H\right)=\chi\left(K_{Y}+H\right), \\
q(X)=q(Y)+h^{1}(-H)=3+h^{1}(-H), \\
p_{g}(X)=p_{g}(Y)+h^{0}\left(K_{Y}+H\right)=1+h^{0}\left(K_{Y}+H\right) .
\end{gathered}
$$

Since $h^{1}(-H)=0$ and $\chi\left(K_{Y}+H\right)=h^{0}\left(K_{Y}+H\right)$ by Kodaira vanishing, we get $q(X)=3$ and $p_{g}(X)-\left(\chi\left(K_{X}\right)+q(X)-1\right)=-1$. By taking $H$ a multiple of a fixed very ample $L \in \operatorname{Pic}(Y)$, one obtains examples with $\chi\left(K_{X}\right)$ arbitrarily large.

In addition, in [4] $Y$ is constructed as a desingularization of a certain $\mathbb{Z}_{2}^{2}$-cover of a product of three elliptic curves and it is easy to see that replacing the elliptic curves by abelian varieties one can obtain examples of varieties $Y$ of any dimension greater than or equal to 3 that are of general type and maximal Albanese dimension and have $p_{g}(Y)=1, \chi\left(K_{Y}\right)=0$ and $q(Y)=\operatorname{dim} Y$. Taking a double cover $X \rightarrow Y$ as above, one obtains examples with $\chi\left(K_{X}\right)$ arbitrarily large and $p_{g}(X)-\left(\chi\left(K_{X}\right)+q(X)-1\right)=$ $2-\operatorname{dim} X<0$.

Example 5.2 (Varieties with Albanese general type fibrations) As in Example 5.1, let $Y$ be a variety of general type and maximal Albanese dimension with $q(Y)=$ $\operatorname{dim} Y=: n \geq 3, p_{g}(Y)=1$ and $\chi\left(K_{Y}\right)=0$. Let $C$ be a curve of genus 2 and let $Z:=Y \times C$; the variety $Z$ is of general type and of Albanese general type, with $\operatorname{dim} Z=n+1, q(Z)=n+2, p_{g}(Z)=2$, hence $p_{g}(Z)-\left(\chi\left(K_{Z}\right)+q(Z)-1\right)=$ $1-n<0$. As in Example 5.1, by taking a double cover $X \rightarrow Z$ branched on a smooth very ample divisor, one obtains examples with $p_{g}(X)-\left(\chi\left(K_{X}\right)+q(X)-1\right)=1-n<0$.

Notice that all these examples have an irrational pencil of genus 2 induced by the second projection $X \rightarrow C$.

Example 5.3 (Varieties with $q(X)=\operatorname{dim} X+1$ ) Let $A$ be an abelian variety of dimension $q$ and let $X \subset A$ be a smooth ample divisor. Using the adjunction formula and Kodaira vanishing, one sees immediately that $q(X)=q=\operatorname{dim} X+1$ and $p_{g}(X)=\chi\left(K_{X}\right)+q-1$, and hence we have that $\left|K_{X}\right|$ is not exorbitant by Theorem 1.2. By [5, Section 3], the same is true also when $X$ is a desingularization of an irreducible theta divisor in a principally polarized abelian variety. In addition, standard computations show that by taking a smooth double cover $X \rightarrow Z$ with ample branch locus where is $Z$ a variety such that $p_{g}(Z)=\chi\left(K_{Z}\right)+q-1$ and $q(Z)=\operatorname{dim} Z+1$, then the Albanese map of $X$ factorizes through the double cover 
$X \rightarrow Z$ and $p_{g}(X)=\chi\left(K_{X}\right)+q-1, q(X)=\operatorname{dim} X+1$. Iterating this construction, one gets examples with $p_{g}=\chi+q-1$ and Albanese map of arbitrarily high degree. Next we describe some examples of $n$-dimensional varieties with $q=n+1$ for which the difference $p_{g}(X)-\left(\chi\left(K_{X}\right)+q-1\right)$ can be arbitrarily large. Let $D$ be a smooth ample divisor in an abelian variety $A$ of dimension $q:=n+1, n \geq 3$, let $Y \subset \mathbb{P}^{n}$ be a smooth hypersurface of degree $d$ and let $X \subset D \times Y$ be an $n$-dimensional general complete intersection of very ample divisors. Since the morphism $X \rightarrow D$ is generically finite, the variety $X$ has maximal Albanese dimension; in addition, by the Lefschetz Theorem, $h^{i}\left(\mathcal{O}_{X}\right)=h^{i}\left(\mathcal{O}_{D \times Y}\right)$ for $i<n$. Using Künneth formula, we get $h^{i}\left(\mathcal{O}_{D \times Y}\right)=h^{i}\left(\mathcal{O}_{D}\right)=\left(\begin{array}{l}q \\ i\end{array}\right), i \leq n-2 ; \quad h^{n-1}\left(\mathcal{O}_{D \times Y}\right)=h^{n-1}\left(\mathcal{O}_{D}\right)+h^{n-1}\left(\mathcal{O}_{Y}\right)$. In particular, we have $q(X)=q=n+1$. It follows that

$$
p_{g}(X)=\chi\left(K_{X}\right)-\sum_{i=1}^{n}(-1)^{i} h^{n-i}\left(\mathcal{O}_{X}\right)=\chi\left(K_{X}\right)+q-1+h^{n-1}\left(\mathcal{O}_{Y}\right) \text {. }
$$

Hence, by taking $d \gg 0$ we can make $p_{g}(X)-\left(\chi\left(K_{X}\right)+q-1\right)=p_{g}(Y)$ arbitrarily large.

We finish by posing a couple of questions.

Question 5.4 It would be interesting to have more geometrical information on varieties with $q(X)=\operatorname{dim} X+1$ and $p_{g}-(\chi+q-1)>0$. For instance, the examples that we know (described in Example 5.3) all have nonbirational Albanese map. One may wonder whether this is true for all varieties with $q=n+1$ and $p_{g}-(\chi+q-1)>0$.

Question 5.5 Can one describe $\left|K_{X}\right| \cap \mathcal{P}_{\text {main }}$ also for varieties of dimension greater than 2 ? For instance, if one could show that $\mathcal{P}_{\text {main }} \cap\left|K_{X}\right|$ is a nondegenerate subvariety of $\left|K_{X}\right|$ then Corollary 1.4 would extend to higher dimension.

\section{References}

[1] M Artin, On the solutions of analytic equations, Invent. Math. 5 (1968) 277-291 MR0232018

[2] A Beauville, Annulation du $H^{1}$ et systèmes paracanoniques sur les surfaces, J. Reine Angew. Math. 388 (1988) 149-157 MR944188

[3] F Catanese, Moduli and classification of irregular Kaehler manifolds (and algebraic varieties) with Albanese general type fibrations, Invent. Math. 104 (1991) 263-289 MR1098610 
[4] J A Chen, C D Hacon, On the irregularity of the image of the Iitaka fibration, Comm. Algebra 32 (2004) 203-215 MR2036231

[5] L Ein, R Lazarsfeld, Singularities of theta divisors and the birational geometry of irregular varieties, J. Amer. Math. Soc. 10 (1997) 243-258 MR1396893

[6] M Green, R Lazarsfeld, Deformation theory, generic vanishing theorems, and some conjectures of Enriques, Catanese and Beauville, Invent. Math. 90 (1987) 389-407 MR910207

[7] M Green, R Lazarsfeld, Higher obstructions to deforming cohomology groups of line bundles, J. Amer. Math. Soc. 4 (1991) 87-103 MR1076513

[8] R Lazarsfeld, M Popa, Derivative complex, BGG correspondence, and numerical inequalities for compact Kähler manifolds, Invent. Math. 182 (2010) 605-633 MR2737707

[9] M Mendes Lopes, R Pardini, G P Pirola, Brill-Noether loci for divisors on irregular varieties, to appear in Journal of European Math. Soc. arXiv:1112.6357

[10] G Pareschi, M Popa, Strong generic vanishing and a higher-dimensional Castelnuovode Franchis inequality, Duke Math. J. 150 (2009) 269-285 MR2569614

[11] Z Ran, On subvarieties of abelian varieties, Invent. Math. 62 (1981) 459-479 MR604839

[12] C Simpson, Subspaces of moduli spaces of rank one local systems, Ann. Sci. École Norm. Sup. 26 (1993) 361-401 MR1222278

Centro de Análise Matemática, Geometria e Sistemas Dinâmicos

Departamento de Matemática, Instituto Superior Técnico, Universidade Técnica de Lisboa Av. Rovisco Pais, 1049-001 Lisboa, Portugal

Dipartimento di Matematica, Università di Pisa

Largo B. Pontecorvo 5, I-56127 Pisa, Italy

Dipartimento di Matematica, Università di Pavia

Via Ferrata 1, I-27100 Pavia, Italy

mmlopes@math.ist.utl.pt, pardini@dm.unipi.it,

gianpietro.pirola@unipv.it

http://www.math.ist.utl.pt/ mmlopes/,

http://www.dm.unipi.it/ pardini/, http://www-dimat.unipv.it/ pirola/

Proposed: Richard Thomas

Received: 25 August 2012

Seconded: Jim Bryan, Ronald Stern

Accepted: 31 January 2013 